\title{
Topologically Invertible Elements and Topological Spectrum
} by

\author{
Mati ABEL and Wiesław ŻELAZKO
}

Presented by Stanistaw KWAPIEŃ

Summary. Properties of topologically invertible elements and the topological spectrum of elements in unital semitopological algebras are studied. It is shown that the inversion $x \mapsto x^{-1}$ is continuous in every invertive Fréchet algebra, and singly generated unital semitopological algebras have continuous characters if and only if the topological spectrum of the generator is non-empty. Several open problems are presented.

\section{Introduction}

1.1. A semitopological algebra is a real or complex linear topological space with an associative separately continuous multiplication. An element $x$ in a semitopological algebra $A$ with unit element $e_{A}$ is said to be left (respectively, right) topologically invertible if $e_{A} \in \overline{A x}$ (respectively, $e_{A} \in \overline{x A}$ ) $\left({ }^{1}\right.$ ), or equivalently, if there is a net $\left(z_{\alpha}\right)_{\alpha \in \mathcal{A}}$ in $A$ such that $\lim _{\alpha} z_{\alpha} x=e_{A}$ (respectively, $\lim _{\alpha} x z_{\alpha}=e_{A}$ ). An element $x$ in $A$ is topologically invertible if it is left and right topologically invertible. The set of all left (respectively, right) invertible elements in $A$ will be denoted by $G_{l}(A)$ (respectively, $G_{r}(A)$ ), and the set of all topologically left (respectively, right) invertible elements in $A$ by $G_{l}^{t}(A)$ (respectively, $G_{r}^{t}(A)$ ). Hence, the set $G(A)$ of all invertible elements equals $G_{l}(A) \cap G_{r}(A)$, and the set $G^{t}(A)$ of all topologically invertible elements equals $G_{l}^{t}(A) \cap G_{r}^{t}(A)$. It is easy to see that $G(A) \subset G^{t}(A)$. In

2000 Mathematics Subject Classification: Primary 46H05; Secondary 46H20.

Key words and phrases: topologically invertible elements, topological spectrum, spectral mapping property, discontinuity of inversion, functional topological spectrum.

Research supported in part by Estonian Science Foundation grant 6205 .

$\left({ }^{1}\right)$ Here and later on $\bar{U}$ denotes the closure of $U$ in $A$. 
particular, when $G(A)=G^{t}(A), A$ is called an invertive algebra $\left({ }^{2}\right)$ (see $[2$, p. 14]); and a topologically invertible element is said to be proper (see [20, p. 323]) if it is non-invertible. Properties of topologically invertible elements have been studied in several papers, for example, in [2], [6]-[9], [11], [13]-[16] and [18]-[20].

1.2. Let $A$ be a semitopological algebra, and $m(A)$ the set of all closed two-sided ideals in $A$ which are maximal as left or right ideals in $A$. A semitopological algebra $A$ over $\mathbb{K}$ is called a Gelfand-Mazur algebra if $A / M$ (in the quotient topology) is topologically isomorphic to $\mathbb{K}$ for each $M \in m(A)$, and a simplicial algebra if every closed regular left (respectively, right) ideal of $A$ is contained in some closed maximal left (respectively, right) ideal of $A$. Main classes of Gelfand-Mazur algebras have been described in [1] or [4]. It is known (see [5, Corollary 5] $\left(^{3}\right)$ ) that every commutative unital locally $m$-pseudoconvex Hausdorff algebra is simplicial.

1.3. Let $A$ be a semitopological algebra over $\mathbb{K}$ with unit element $e_{A}$, $\mathfrak{M}(A)$ the set of all continuous $\mathbb{K}$-valued characters (non-zero multiplicative linear functionals) on $A$,

$$
\sigma_{A}(x)=\left\{\lambda \in \mathbb{K}: x-\lambda e_{A} \notin G(A)\right\}
$$

the (algebraic) spectrum of $x \in A$, and

$$
\varrho_{A}(x)=\sup \left\{|\lambda|: \lambda \in \sigma_{A}(x)\right\}
$$

the (algebraic) spectral radius of $x$. The (algebraic) spectrum $\sigma_{A}(x)$ and the spectral radius $\varrho_{A}(x)$ of $x$ play an important role in invertive algebras. For non-invertive algebras a similar role is played by the topological spectrum

$$
\sigma_{A}^{t}(x)=\left\{\lambda \in \mathbb{K}: x-\lambda e_{A} \notin G^{t}(A)\right\}
$$

and the topological spectral radius

$$
\varrho^{t}(x)=\sup \left\{|\lambda|: \lambda \in \sigma_{A}^{t}(x)\right\} .
$$

Similarly to the spectral radius, we set $\varrho_{A}^{t}(x)=0$ if $\sigma_{A}^{t}(x)=\emptyset$, and $\varrho_{A}^{t}(x)=\infty$ if $\sigma_{A}^{t}(x)$ is unbounded. It is easy to see that $\sigma_{A}^{t}(x) \subset \sigma_{A}(x)$ and $\varrho_{A}^{t}(x) \leq$ $\varrho_{A}(x)$ for each $x \in A$. Moreover, $A$ is invertive if and only if $\sigma_{A}^{t}(x)=\sigma_{A}(x)$ for each $a \notin G(A)$. Indeed, if $A$ is invertive, then $\sigma_{A}^{t}(x)=\sigma_{A}(x)$ for each $a \in A$. Conversely, let $a \in A \backslash G(A)$. Then $0 \in \sigma_{A}(a)$. If now $\sigma_{A}(a)=\sigma_{A}^{t}(a)$, then $a \notin G^{t}(A)$. Hence, $G(A)=G^{t}(A)$, and so $A$ is invertive.

$\left({ }^{2}\right)$ It is known (see [2, Corollary 2]) that every complete unital locally $m$-pseudoconvex algebra is an invertive algebra, but any commutative unital $F$-algebra with a discontinuous inverse is not (see [19, Proposition 4]).

$\left({ }^{3}\right)$ For complete algebras see [3, Proposition 2] or [10, Corollary 7.1.14], and for locally $m$-convex Hausdorff algebras see [12, pp. 321-322]. 
Let $x \in A, \varphi \in \mathfrak{M}(A)$ and $x-\varphi(x) e_{A} \in G^{t}(A)$. Then there are nets $\left(x_{\alpha}\right)_{\alpha \in \mathcal{A}}$ and $\left(y_{\mu}\right)_{\mu \in \mathcal{M}}$ in $A$ such that both $\left(\left(x-\varphi(x) e_{A}\right) x_{\alpha}\right)_{\alpha \in \mathcal{A}}$ and $\left(y_{\mu}\left(x-\varphi(x) e_{A}\right)\right)_{\mu \in \mathcal{M}}$ converge to $e_{A}$. Since $\varphi$ is continuous, the nets $\left(\varphi\left(x-\varphi(x) e_{A}\right) \varphi\left(x_{\alpha}\right)\right)_{\alpha \in \mathcal{A}}$ and $\left(\varphi\left(x-\varphi(x) e_{A}\right) \varphi\left(y_{\mu}\right)\right)_{\mu \in \mathcal{M}}$ then converge to 1 , but this is not possible, because $\varphi\left(x-\varphi(x) e_{A}\right)=0$. Hence, $x-\varphi(x) e_{A} \notin$ $G^{t}(A)$ for each $\varphi \in \mathfrak{M}(A)$. Consequently,

$$
\{\varphi(x): \varphi \in \mathfrak{M}(A)\} \subset \sigma_{A}^{t}(x)
$$

for each $x \in A$. In particular, if $\mathfrak{M}(A)$ is not empty and

$$
\sigma_{A}^{t}(x)=\{\varphi(x): \varphi \in \mathfrak{M}(A)\}
$$

for each $x \in A$, we will say that $x$ has functional topological spectrum.

1.4. In this paper, we will study the properties of topologically invertible elements and of the topological spectrum of elements in unital semitopological algebras. Among other results, it is shown that (a) the set of all topologically invertible elements in any unital Fréchet (that is, complete and metrizable) algebra is a $G_{\delta^{-}}$set; (b) if the inversion in a unital Fréchet algebra $A$ is discontinuous, then $A$ has left and right dense ideals; (c) if $A$ is a commutative complex unital semitopological algebra, then the topological spectrum has the spectral mapping property; (d) every element of a commutative unital simplicial Gelfand-Mazur algebra has functional topological spectrum; and (e) if every element in a unital semitopological algebra $A$ has functional topological spectrum, then the topological spectral radius is a submultiplicative seminorm on $A$. Several open problems are presented at the end of paper.

2. Properties of $G^{t}(A)$ for a Fréchet algebra $A$. Let $A$ be a unital (real or complex) non-commutative Fréchet algebra with $F$-norm $\|\cdot\|$, i.e. with a function $x \mapsto\|x\|$ on $A$ such that

(i) $\|x\| \geq 0$ for each $x \in A$, and $\|x\|=0$ if and only if $x=\theta_{A}\left({ }^{4}\right)$;

(ii) $\|x+y\| \leq\|x\|+\|y\|$ for all $x, y \in A$;

(iii) $(\lambda, x) \mapsto\|\lambda x\|$ is a jointly continuous map from $\mathbb{K} \times A$ to $\mathbb{R}_{+}$.

Let $\left\|e_{A}\right\|=1$, and

$$
g_{l}(x)=\inf _{u \in A}\left\|u x-e_{A}\right\| \quad \text { and } \quad g_{r}(x)=\inf _{u \in A}\left\|x u-e_{A}\right\|
$$

for each $x \in A$. Then

$$
G_{l}^{t}(A)=\left\{x \in A: g_{l}(x)=0\right\} \quad \text { and } \quad G_{r}^{t}(A)=\left\{x \in A: g_{r}(x)=0\right\} .
$$

$\left({ }^{4}\right)$ Here and below, $\theta_{A}$ denotes the null element of $A$. 
LEMma 1. Let $A$ be a unital non-commutative Fréchet algebra. Then the sets

$$
S_{l, \lambda}=\left\{x \in A: g_{l}(x)<\lambda\right\} \quad \text { and } \quad S_{r, \lambda}=\left\{x \in A: g_{r}(x)<\lambda\right\}
$$

are open in $A$ for any $\lambda>0$.

Proof. The proof is similar to the proof of Lemma 1 in [19].

Corollary 1. The function $g_{l}$ is continuous at all points of $G_{l}^{t}(A)$, and $g_{r}$ is continuous at all points of $G_{r}^{t}(A)$.

It is known (see [19, Proposition 3]) that $G^{t}(A)$ is a $G_{\delta^{-}}$set for any commutative unital $F$-algebra $A$. In the non-commutative case, we have

Proposition 1. Let $A$ be a non-commutative unital F-algebra. Then $G_{l}^{t}(A), G_{r}^{t}(A)$ and $G^{t}(A)$ are $G_{\delta}$-sets.

Proof. Since

$$
G_{l}^{t}(A)=\bigcap_{n \in \mathbb{N}}\left\{x \in A: g_{l}(x)<1 / n\right\}, \quad G_{r}^{t}(A)=\bigcap_{n \in \mathbb{N}}\left\{x \in A: g_{r}(x)<1 / n\right\},
$$

$G_{l}^{t}(A)$ and $G_{r}^{t}(A)$ are $G_{\delta^{-}}$sets by Lemma 1 . Therefore so is $G^{t}(A)$ as the intersection of two $G_{\delta}$-sets.

Corollary 2. Let $A$ be a unital F-algebra. If the inversion $x \mapsto x^{-1}$ is discontinuous in $A$, then $G^{t}(A) \backslash G(A) \neq \emptyset$.

Proof. It is known (see, for example, [17, Theorem 1.6]) that the inversion is continuous in $A$ if and only if $G(A)$ is a $G_{\delta^{-}}$set and $G(A) \subset G^{t}(A)$. If the inversion is discontinuous, then $G^{t}(A) \backslash G(A) \neq \emptyset$ by Proposition 1.

COROLlary 3. In every invertive Fréchet algebra the inversion is continuous.

COROLlary 4. If the inversion is discontinuous in a unital F-algebra A, then $A$ has a dense left and a dense right ideal.

Proof. By Corollary 2 there is an element

$$
x \in\left(G_{l}^{t}(A) \cap G_{r}^{t}(A)\right) \backslash G(A) .
$$

Suppose that $x \in G_{l}(A)$. Then there exists $a \in A$ such that $a x=e_{A}$. Since $x \in G_{r}^{t}(A)$ as well, there is a sequence $\left(x_{n}\right) \subset A$ such that $\left(x x_{n}\right)$ converges to $e_{A}$. This implies that $\left(x_{n}\right)$ converges to $a$ and $x a=e_{A}$. Thus $x \in G(A)$, which is not the case. Similarly, from $x \in G_{r}(A)$ it follows that $x \in G(A)$, which is again impossible. Consequently,

$$
x \in\left(G_{l}^{t}(A) \backslash G_{l}(A)\right) \cap\left(G_{r}^{t}(A) \backslash G_{r}(A)\right) .
$$

This means that the left ideal $A x$ and the right ideal $x A$ are dense in $A$. 
3. Singly generated topological algebras. We say that a semitopological algebra $A$ is singly generated by an element $x_{0} \in A$ (the generator) if the set of all polynomials in $x_{0}$ (with real coefficients in the case of a real algebra) is dense in $A$. We now show how the topological spectrum can be used to decide when a unital singly generated semitopological algebra $A$ has continuous characters $\left({ }^{5}\right)$. In this case $A$ is commutative.

Proposition 2. Let $A$ be a unital singly generated (real or complex) semitopological algebra and $x_{0}$ the generator of $A$. Then there is a one-to-one correspondence between the elements in $\mathfrak{M}(A)$ and the scalars in $\sigma_{A}^{t}\left(x_{0}\right)$.

Proof. Suppose that $\sigma_{A}^{t}\left(x_{0}\right) \neq \emptyset$ and fix $\lambda \in \sigma_{A}^{t}\left(x_{0}\right)$. Let $A_{0}$ be the subalgebra of $A$ consisting of all polynomials $p$ in $x_{0}$ (then $A_{0}$ is a dense subalgebra of $A$ ) and let

$$
f_{\lambda}\left(p\left(x_{0}\right)\right)=p(\lambda)
$$

We show that $f_{\lambda}$ is a character on $A_{0}$. To this end, we first have to show that $f_{\lambda}$ is well defined, i.e. if $p_{1}\left(x_{0}\right)=p_{2}\left(x_{0}\right)$, then $p_{1}(\lambda)=p_{2}(\lambda)$, or that $p(x)=0$ implies $p(\lambda)=0$. If this is not so, i.e. if $p\left(x_{0}\right)=0$ but $p(\lambda) \neq 0$, then the relation

$$
p\left(x_{0}\right)-p(\lambda) e_{A}=\left(x_{0}-\lambda e_{A}\right) q\left(x_{0}, \lambda\right)
$$

for some polynomial $q$ in two variables implies that

$$
e_{A}=-[p(\lambda)]^{-1}\left(x_{0}-\lambda e_{A}\right) q\left(x_{0}, \lambda\right),
$$

i.e. $\lambda$ is not in the (algebraic) spectrum $\sigma_{A}\left(x_{0}\right)$. So, $\lambda$ cannot be in the (smaller) spectrum $\sigma_{A}^{t}\left(x_{0}\right)$, which gives a contradiction. Hence, the formula (a) defines a multiplicative linear functional on $A_{0}$.

We now show that $f_{\lambda}$ is continuous. Indeed, if not, then $f_{\lambda}^{-1}(0)$ is dense in $A_{0}$, and so there is a net $\left(p_{\alpha}\right)_{\alpha \in \mathcal{A}} \subset f_{\lambda}^{-1}(0)$ such that $\left(p_{\alpha}\left(x_{0}\right)\right)_{\alpha \in \mathcal{A}}$ tends to $e_{A}$. Since

$$
p_{\alpha}\left(x_{0}\right)=\left(x_{0}-\lambda e_{A}\right) q_{\alpha}\left(x_{0}, \lambda\right)=r_{\alpha}\left(x_{0}, \lambda\right)\left(x_{0}-\lambda e_{A}\right)
$$

for some polynomials $q_{\alpha}$ and $r_{\alpha}$ in two variables, $x_{0}-\lambda e_{A}$ is topologically invertible, which is a contradiction, since $\lambda \in \sigma_{A}^{t}\left(x_{0}\right)$. Thus, $f_{\lambda}$ is a continuous character on $A_{0}$ and extends by continuity to an element of $\mathfrak{M}(A)$, which we also denote by $f_{\lambda}$. Then

$$
f_{\lambda}\left(x_{0}\right)=\lambda
$$

so that to different scalars $\lambda$ correspond different functionals in $\mathfrak{M}(A)$.

Conversely, assume that $\mathfrak{M}(A) \neq \emptyset$, let $f \in \mathfrak{M}(A)$, and put $\lambda=f\left(x_{0}\right)$. Then $x=x_{0}-\lambda e_{A} \in f^{-1}(0)$ and $x$ is not topologically invertible, as other-

$\left({ }^{5}\right)$ This result has been extended by the second author to finitely generated commutative topological algebras (see [21]). 
wise there is a net $\left(u_{\alpha}\right)_{\alpha \in \mathcal{A}}$ with $\left(u_{\alpha} x\right)_{\alpha \in \mathcal{A}} \rightarrow e_{A}$, which is impossible (because then $0=f\left(u_{\alpha} x\right) \rightarrow f\left(e_{A}\right)=1$ for each $\left.\alpha \in \mathcal{A}\right)$. Thus $\lambda \in \sigma_{A}^{t}\left(x_{0}\right)$ and the conclusion follows (the announced one-to-one correspondence is given by (b)).

Thus we have a situation somewhat similar to that of singly generated (unital) Banach algebras, where the spectrum of the generator can be identified with the maximal ideal space (equipped with the Gelfand topology) both as a set and as a compact space. The example below shows that the two sets may not be homeomorphic in general.

ExAmPle. Let $A$ be the Cartesian product of continuum many copies of the field of scalars, provided with the coordinatewise algebra operations and the product topology. We can identify $A$ with the algebra of all scalar-valued functions on the complex plane $\mathbb{C}$ with the topology of pointwise convergence. This topology is given by the seminorms $\|x\|_{S}=\max _{\zeta \in S}|x(\zeta)|$ for each $x \in A$, where $S$ runs over the family $\mathcal{F}$ of all finite subsets of $\mathbb{C}$. Clearly, $A$ is a complete multiplicatively convex algebra, and all its multiplicative linear functionals are continuous and are of the form $f_{\zeta}(x)=x(\zeta)$, where $\zeta \in \mathbb{C}$ (see, e.g., [14]). The algebra $A$ is singly generated by the fuction $x_{0}$ defined by $x_{0}(\zeta)=\zeta$. In fact, for any $x$ in $A$ and $S$ in $\mathcal{F}$, there is a polynomial $p$ with scalar coefficients such that $p(x)(\zeta)=x(\zeta)$ for all $\zeta$ in $S$. Thus $\|p(x)-x\|_{S}=0$ and $x$ can be approximated by polynomials of $x$. We can identify $\mathfrak{M}(A)$ with $\mathbb{C}$ as a set, but, as shown below, not as a topological space, because the Gelfand topology of $\mathfrak{M}(A)$ is discrete. To show this, consider the element $x_{1}$ of $A$ given by

$$
x_{1}(\zeta)= \begin{cases}1 & \text { if } \zeta=0 \\ 0 & \text { otherwise }\end{cases}
$$

and the character $f_{0}(x)=x(0)$. Its neighbourhood

$$
U\left(f ; x_{1}, 1 / 2\right)=\left\{f \in \mathfrak{M}(A):\left|f\left(x_{1}\right)-f_{0}\left(x_{1}\right)\right|<1 / 2\right\}
$$

in the Gelfand topology consists of $f_{0}$ only, and our claim follows.

As a corollary to Proposition 2 we obtain the following

Proposition 3. A singly generated unital semitopological (real or complex) algebra has a continuous character if and only if the topological spectrum of its generator is non-empty.

REMARK. The above result fails to be true if we replace the topological spectrum by the algebraic spectrum. The $B_{0}$-algebra $L^{\omega}[0,1]$ of Arens (see [8] or [20, Example 10.5]), equal to $\bigcap_{p=1}^{\infty} L_{p}[0,1]$, is singly generated $\left({ }^{6}\right)$ by the function $x_{0}$, where $x_{0}(t)=t$ for each $t \in \mathbb{C}$ (that is, the subalgebra of

$\left({ }^{6}\right)$ All algebraic operations in $L^{\omega}[0,1]$ are defined pointwise. 
all polynomials in $x_{0}$ is dense in $\left.L^{\omega}[0,1]\right)$. Then $\sigma_{L^{\omega}[0,1]}\left(x_{0}\right)=[0,1] \neq \emptyset$. On the other hand (see [20, Subsection 14.2]), $L^{\omega}[0,1]$ has no characters and so no continuous characters. It can be easily seen that the translates $x_{0}-\lambda e$ with $0 \leq \lambda \leq 1$ are not invertible and not topologically invertible. Therefore, $\sigma_{L^{\omega}[0,1]}^{t}\left(x_{0}\right)=\emptyset$ (this also follows from Proposition 3$)$.

\section{Topological spectrum}

4.1. Properties of the topological spectrum. Let $A$ be a unital semitopological algebra. The topological spectrum of elements of $A$ has several properties similar to the (algebraic) spectrum. It is easy to see that $\sigma_{A}^{t}\left(\theta_{A}\right)=\{0\}$, $\sigma_{A}^{t}\left(e_{A}\right)=\{1\}$ and $\sigma_{A}^{t}(e)=\{0,1\}$ for any idempotent $e$ of $A$ with $e \neq \theta_{A}$ and $e \neq e_{A}$. Moreover, we have

Proposition 4. Let $A$ be a unital semitopological algebra. For any $x, y$ $\in A$ with $\sigma_{A}^{t}(x) \neq \emptyset$ and $\sigma_{A}^{t}(x y) \neq \emptyset$ and $\mu \in \mathbb{K}$ we have

(a) $\sigma_{A}^{t}(\mu x)=\mu \sigma_{A}^{t}(x)$;

(b) $\sigma_{A}^{t}\left(\mu e_{A}+x\right)=\mu+\sigma_{A}^{t}(x)$;

(c) if $x \in G(A)$, then $\sigma_{A}^{t}\left(x^{-1}\right)=\left\{\lambda^{-1}: \lambda \in \sigma_{A}^{t}(x)\right\}$;

(d) $\sigma_{A}^{t}(x y) \cup\{0\}=\sigma_{A}^{t}(y x) \cup\{0\}$.

Proof. Since $\mu x-\lambda e_{A}=\mu\left(x-\mu^{-1} \lambda e_{A}\right)$ for all $\mu \neq 0$ and $\lambda \in \mathbb{K}$, it follows that $\lambda \in \sigma_{A}^{t}(\mu x)$ if and only if $\mu^{-1} \lambda \in \sigma_{A}^{t}(x)$. Therefore

$$
\sigma_{A}^{t}(\mu x) \subset \mu \sigma_{A}^{t}(x)=\mu \sigma_{A}^{t}\left(\mu^{-1}(\mu x)\right) \subset \sigma_{A}^{t}(\mu x)
$$

for $\mu \neq 0$. Thus, we have (a) for $\mu \neq 0$ (if $\mu=0$, then (a) also holds). Similarly to (a), the equality (b) follows from the identity

$$
\left(\mu e_{A}+x\right)-\lambda e_{A}=x-(\lambda-\mu) e_{A},
$$

and (c) follows from

$$
x^{-1}-\lambda^{-1} e_{A}=\left(-\lambda^{-1} x^{-1}\right)\left(x-\lambda e_{A}\right)=\left(x-\lambda e_{A}\right)\left(-\lambda^{-1} x^{-1}\right),
$$

because $\left(-\lambda^{-1} x^{-1}\right)\left(x-\lambda e_{A}\right)=\left(x-\lambda e_{A}\right)\left(-\lambda^{-1} x^{-1}\right)$ for each $x \in G(A)$.

To prove (d), we first show that $e_{A}-x y \in G^{t}(A)$ if and only if $e_{A}-y x \in$ $G^{t}(A)$. Let $e_{A}-x y \in G^{t}(A)$. Then there exist nets $\left(u_{\alpha}\right)_{\alpha \in \mathcal{A}}$ and $\left(v_{\beta}\right)_{\beta \in \mathcal{B}}$ in $A$ such that $\left(\left(e_{A}-x y\right) u_{\alpha}\right)_{\alpha \in \mathcal{A}}$ and $\left(v_{\beta}\left(e_{A}-x y\right)\right)_{\beta \in \mathcal{B}}$ converge to $e_{A}$. Since

$$
\left(y u x+e_{A}\right)\left(e_{A}-y x\right)=y\left[u\left(e_{A}-x y\right)\right] x+e_{A}-y x
$$

and

$$
\left(e_{A}-y x\right)\left(y u x+e_{A}\right)=y\left[\left(e_{A}-x y\right) u\right] x+e_{A}-y x
$$

for all $x, y, u \in A$ and the multiplication in $A$ is separately continuous, the nets $\left(\left(y v_{\beta} x+e_{A}\right)\left(e_{A}-y x\right)\right)_{\beta \in \mathcal{B}}$ and $\left(\left(e_{A}-y x\right)\left(y u_{\alpha} a+e_{A}\right)\right)_{\alpha \in \mathcal{A}}$ converge to $e_{A}$. Hence, $e_{A}-y x \in G^{t}(A)$. By symmetry, $e_{A}-y x \in G^{t}(A)$ implies $e_{A}-x y \in G^{t}(A)$. 
Let now $\lambda \in \sigma_{A}^{t}(x y)$. Then $x y-\lambda e_{A} \notin G^{t}(A)$. Since

$$
x y-\lambda e_{A}=-\lambda\left(e_{A}-\left(\lambda^{-1} x\right) y\right)
$$

for $\lambda \neq 0$, we have $e_{A}-\left(\lambda^{-1} y\right) x \notin G^{t}(A)$. Hence, $\lambda \in \sigma_{A}^{t}(y x)$. Thus

$$
\sigma_{A}^{t}(x y) \cup\{0\} \subset \sigma_{A}^{t}(y x) \cup\{0\} .
$$

Interchanging $x$ and $y$, we get the reverse inclusion.

Lemma 2. Let $A$ and $B$ be unital semitopological algebras and $\pi$ a continuous homomorphism from $A$ into $B$ such that $\pi\left(e_{A}\right)=e_{B}$. Then

$$
\sigma_{B}^{t}(\pi(x)) \subset \sigma_{A}^{t}(x)
$$

for all $x \in A$.

Proof. Let $\lambda \in \sigma_{B}^{t}(\pi(x))$. Then $\pi\left(x-\lambda e_{A}\right)=\pi(x)-\lambda e_{B} \notin G^{t}(B)$. Therefore $x-\lambda e_{A} \notin G^{t}(A)$. Thus $\lambda \in \sigma_{A}^{t}(x)$.

Proposition 5. Let $A$ and $B$ be unital semitopological algebras and $\pi$ a continuous open homomorphism from $A$ onto $B$. If there exists a neighbourhood $O$ of zero in $A$ such that $e_{A}+O+\operatorname{ker} \pi \subset G^{t}(A)$, then

$$
\sigma_{B}^{t}(\pi(x))=\sigma_{A}^{t}(x)
$$

for each $x \in A$.

Proof. By Lemma 2, the inclusion $\sigma_{B}^{t}(\pi(x)) \subset \sigma_{A}^{t}(x)$ holds. To prove the opposite inclusion, assume that $\lambda \notin \sigma_{B}^{t}(\pi(x))$ and $O$ is as in the statement. Then $\pi(x)-\lambda e_{B} \in G^{t}(B)$. Therefore there exist nets $\left(x_{\mu}\right)_{\mu \in M}$ and $\left(y_{\nu}\right)_{\nu \in V}$ in $A$ such that the nets $\left(\left(\pi(x)-\lambda e_{B}\right) \pi\left(x_{\mu}\right)\right)_{\mu \in M}$ and $\left(\pi\left(y_{\nu}\right)\left(\pi(x)-\lambda e_{B}\right)\right)_{\nu \in V}$ converge to $e_{B}$ in $B$. Since $\pi$ is open, $\pi(O)$ is a neighbourhood of zero in $B$. Hence there exist $\mu_{0} \in M$ and $\nu_{0} \in V$ such that

$$
\left(x-\lambda e_{A}\right) x_{\mu}-e_{A} \in \pi^{-1}(\pi(O))=O+\operatorname{ker} \pi
$$

whenever $\mu>\mu_{0}$, and

$$
y_{\nu}\left(x-\lambda e_{A}\right)-e_{A} \in \pi^{-1}(\pi(O))=O+\operatorname{ker} \pi
$$

whenever $\nu>\nu_{0}$. This means that

$$
\left(x-\lambda e_{A}\right) x_{\mu} \in e_{A}+O+\operatorname{ker} \pi \subset G^{t}(A)
$$

whenever $\mu>\mu_{0}$ and

$$
y_{\nu}\left(x-\lambda e_{A}\right) \in e_{A}+O+\operatorname{ker} \pi \subset G^{t}(A)
$$

whenever $\nu>\nu_{0}$.

Now we fix $\mu_{1}>\mu_{0}$ and $\nu_{1}>\nu_{0}$. Then there exist nets $\left(u_{\alpha}\right)_{\alpha \in \mathcal{A}}$ and $\left(v_{\beta}\right)_{\beta \in \mathcal{B}}$ in $A$ such that $\left(\left(x-\lambda e_{A}\right)\left(x_{\mu_{1}} u_{\alpha}\right)\right)_{\alpha \in \mathcal{A}}$ and $\left(\left(v_{\beta} y_{\nu_{1}}\right)\left(x-\lambda e_{A}\right)\right)_{\beta \in \mathcal{B}}$ converge to $e_{A}$ in $A$. Consequently, $x-\lambda e_{A} \in G^{t}(A)$, i.e. $\lambda \notin \sigma_{A}^{t}(x)$. Thus, $\sigma_{A}^{t}(x) \subset \sigma_{B}^{t}(\pi(x))$ holds as well. 
Corollary 5. Let $A$ be a unital semitopological algebra, I a two-sided ideal of $A$ and $\pi$ the canonical homomorphism from $A$ onto $A / I$. If there exists a neighbourhood $O$ of zero in $A$ such that $e_{A}+O+I \subset G^{t}(A)$, then

$$
\sigma_{A / I}^{t}(\pi(x))=\sigma_{A}^{t}(x)
$$

for each $x \in A$.

Corollary 6. Let $A$ and $B$ be unital semitopological algebras and $\pi$ a homomorphism and homeomorphism from $A$ into $B$ such that $\pi\left(e_{A}\right)=e_{B}$. If

(a) $G^{t}(A)$ is open in $A$

or

(b) $G^{t}(\pi(A))=G^{t}(B) \cap \pi(A)$,

then

$$
\sigma_{B}^{t}(\pi(x))=\sigma_{A}^{t}(x)
$$

for each $x \in A$.

Proof. The inclusion $\sigma_{B}^{t}(\pi(x)) \subset \sigma_{A}^{t}(x)$ holds for each $x \in A$, by Lemma 2 . If $A$ satisfies condition (a) and $\pi$ is one-to-one, then the opposite inclusion also holds for each $x \in A$, by Proposition 5. Let now $A$ and $\pi$ satisfy condition (b). Let $x \in A$ and $\lambda \notin \sigma_{B}^{t}(\pi(x))$. Then $\pi\left(x-\lambda e_{A}\right) \in G^{t}(\pi(A))$ by (b). Therefore there exist nets $\left(x_{\mu}\right)_{\mu \in M}$ and $\left(y_{\mu}\right)_{\nu \in V}$ in $A$ such that both $\left(\pi\left[\left(x-\lambda e_{A}\right) x_{\mu}\right]\right)_{\mu \in M}$ and $\left(\pi\left[y_{\nu}\left(x-\lambda e_{A}\right)\right]\right)_{\nu \in V}$ converge to $\pi\left(e_{A}\right)$ in $\pi(A)$. Since $\pi^{-1}$ is continuous, $\left(\left(x-\lambda e_{A}\right) x_{\mu}\right)_{\mu \in M}$ and $\left(y_{\nu}\left(x-\lambda e_{A}\right)\right)_{\nu \in V}$ converge to $e_{A}$ in $A$. Hence, $x-\lambda e_{A} \in G^{t}(A)$, i.e. $\lambda \notin \sigma_{A}^{t}(x)$. Consequently, $\sigma_{A}^{t}(x) \subset \sigma_{B}^{t}(\pi(x))$.

Proposition 6. Let $A$ and $B$ be unital semitopological algebras and $\pi$ a continuous homomorphism from $A$ into $B$. If $B$ is a Hausdorff space and $\pi(A)$ is dense in $B$, then

$$
G^{t}(\pi(A))=G^{t}(B) \cap \pi(A) .
$$

Proof. It is easy to see that $G^{t}(\pi(A)) \subset G^{t}(B) \cap \pi(A)$. To prove the opposite inclusion, let $O$ be a neighbourhood of zero in $\pi(A)$ (then there is a neighbourhood $O^{\prime}$ of zero in $B$ with $\left.O=O^{\prime} \cap \pi(A)\right)$ and let $y \in G^{t}(B) \cap \pi(A)$. Then there are $x \in A$ such that $y=\pi(x)$ and a neighbourhood $O_{1}$ of zero in $B$ such that $y O_{1}+O_{1}, O_{1} y+O_{1} \subset O$. Since $y \in G^{t}(B)$, there exist nets $\left(y_{\mu}\right)_{\mu \in M}$ and $\left(z_{\mu}\right)_{\nu \in V}$ in $B$ such that $\left(y y_{\mu}\right)_{\mu \in M}$ and $\left(z_{\nu} y\right)_{\nu \in V}$ converge to $e_{B}$. Therefore, there are $\mu_{0} \in M$ and $\nu_{0} \in V$ such that $y y_{\mu}-e_{B} \in O_{1}$ whenever $\mu>\mu_{0}$, and $z_{\nu} y-e_{B} \in O_{1}$ whenever $\nu>\nu_{0}$. Let $\mu_{1} \in M$ and $\nu_{1} \in V$ be such that $\mu_{1}>\mu_{0}$ and $\nu_{1}>\nu_{0}$. Then $y y_{\mu_{1}}-e_{B} \in O_{1}$ and $z_{\nu_{1}} y-e_{B} \in O_{1}$. Since $\pi(A)$ is dense in $B$, there are nets $\left(u_{\alpha}\right)_{\alpha \in \mathcal{A}}$ and $\left(v_{\beta}\right)_{\beta \in \mathcal{B}}$ such that $\left(\pi\left(u_{\alpha}\right)\right)_{\alpha \in \mathcal{A}}$ converges to $y_{\mu_{1}}$ and $\left(\pi\left(v_{\beta}\right)\right)_{\beta \in \mathcal{B}}$ converges to $z_{\nu_{1}}$. Hence, there 
are $\lambda_{0} \in \mathcal{A}$ and $\beta_{0} \in \mathcal{B}$ such that $\pi\left(u_{\alpha}\right)-y_{\mu_{1}} \in O_{1}$ whenever $\alpha>\alpha_{0}$, and $\pi\left(v_{\beta}\right)-z_{\nu_{1}} \in O_{1}$ whenever $\beta>\beta_{0}$. Taking this into account $\left({ }^{7}\right)$,

$$
\pi(x) \pi\left(u_{\alpha}\right)-\pi\left(e_{A}\right)=\pi(x)\left[\pi\left(u_{\alpha}\right)-y_{\mu_{1}}\right]+\pi(x) y_{\mu_{1}}-e_{B} \in y O_{1}+O_{1} \subset O^{\prime}
$$

whenever $\alpha>\alpha_{0}$, and

$$
\pi\left(v_{\beta}\right) \pi(x)-\pi\left(e_{A}\right)=\left[\pi\left(v_{\beta}\right)-z_{\nu_{1}}\right] \pi(x)+z_{\nu_{1}} \pi(x)-e_{B} \in O_{1} y+O_{1} \subset O^{\prime}
$$

whenever $\beta>\beta_{0}$. Consequently, $\left(\pi(x) \pi\left(u_{\alpha}\right)\right)_{\alpha \in \mathcal{A}}$ and $\left(\pi\left(v_{\beta}\right) \pi(x)\right)_{\beta \in \mathcal{B}}$ converge to $\pi\left(e_{A}\right)$ in $\pi(A)$. Thus, $y \in G^{t}(\pi(A))$.

Corollary 7. Let $A$ and $B$ be unital semitopological algebras and $\pi$ a continuous homomorphism from $A$ into $B$. If $B$ is a Hausdorff space and $\pi(A)$ is dense in $B$, then

$$
\sigma_{\pi(A)}^{t}(\pi(a))=\sigma_{B}^{t}(\pi(a))
$$

for each $a \in A$.

Proof. Let $a \in A$. If $\lambda \in \sigma_{\pi(A)}^{t}(\pi(a))$, then $\pi(a)-\lambda \pi\left(e_{A}\right) \notin G^{t}(\pi(A))$. Hence, $\pi(a)-\lambda \pi\left(e_{A}\right) \notin G^{t}(B)$, by Proposition 6. Therefore, $\lambda \in \sigma_{B}^{t}(\pi(a))$. Thus,

$$
\sigma_{\pi(A)}^{t}(\pi(a)) \subset \sigma_{B}^{t}(\pi(a)) .
$$

The opposite inclusion holds similarly, by Proposition 6 .

COROLlary 8. Let A be a unital semitopological Hausdorff algebra, $\widetilde{A}$ the completion of $A$ and $\tau$ the homeomorphism from $A$ into $\widetilde{A}$ defined by the completion of $A$. Then

$$
\sigma_{\widetilde{A}}^{t}(\tau(a))=\sigma_{A}^{t}(a)
$$

for each $a \in A\left({ }^{8}\right)$.

Proof. Since $\tau(A)$ is dense in $\widetilde{A}$, the assertion holds by Corollary 6 .

4.2. Spectral mapping property for the topological spectrum. To prove the spectral mapping theorem for the topological spectrum, we need the following

Lemma 3. Let $A$ be a commutative unital semitopological algebra, $n \in \mathbb{N}$ and $x_{1}, \ldots, x_{n} \in A$. Then $x_{1} \cdots x_{n} \in G^{t}(A)$ if and only if $x_{i} \in G^{t}(A)$ for each $i \in \mathbb{N}_{n}\left({ }^{9}\right)$.

$\left({ }^{7}\right)$ Since $B$ is a Hausdorff space and $\pi$ is continuous, and $\pi(A)$ is dense in $B$, we have $\pi\left(e_{A}\right)=e_{B}$.

$\left({ }^{8}\right)$ For unital complex metrizable algebras this result is known (see [19, Proposition 16]).

$\left({ }^{9}\right)$ Here and below, $\mathbb{N}_{n}=\{1,2, \ldots, n\}$ for each $n \in \mathbb{N}$. 
Proof. Let $x=x_{1} \cdots x_{n} \in G^{t}(A)$. Then there exists a net $\left(z_{\alpha}\right)_{\alpha \in \mathcal{A}}$ in $A$ such that $\left(x z_{\alpha}\right)_{\alpha \in \mathcal{A}}$ converges to $e_{A}$. Therefore

$$
\left(x_{i}\left(x_{1} \cdots x_{i-1} \cdot x_{i+1} \cdots x_{n} z_{\alpha}\right)\right)_{\alpha \in \mathcal{A}}
$$

converges to $e_{A}$ for each $i \in \mathbb{N}_{n}$. Now for each fixed $\alpha \in \mathcal{A}$ we put $w_{\alpha}^{1}=$ $x_{2} \cdots x_{n} z_{\alpha}, w_{\alpha}^{i}=x_{1} \cdots x_{i-1} \cdot x_{i+1} \cdots x_{n} z_{\alpha}$ for each $i$ with $2 \leq i \leq n-2$ and $w_{\alpha}^{n}=x_{1} \cdots x_{n-1} z_{\alpha}$. Then for any $i \in \mathbb{N}_{n}$ the net $\left(x_{i} w_{\alpha}^{i}\right)_{\alpha \in \mathcal{A}}$ converges to $e_{A}$. Hence, $x_{i} \in G^{t}(A)$ for $1 \leq i \leq n$.

Conversely, let $x_{i} \in G^{t}(A)$ for any $1 \leq i \leq n$. Using induction, it is sufficient to consider the case $n=2$. Since $x_{1} \in G^{t}(A)$, there exists a net $\left(z_{\alpha}\right)_{\alpha \in \mathcal{A}}$ in $A$ such that $\left(x_{1} z_{\alpha}\right)_{\alpha \in \mathcal{A}}$ converges to $e_{A}$, so for any (open) neighbourhood $U$ of zero in $A$ there is $\alpha_{0} \in \mathcal{A}$ such that $x_{1} z_{\alpha} \in e_{A}+U$ whenever $\alpha \geq \alpha_{0}$. Moreover, since $x_{2} \in G^{t}(A)$, there exists a net $\left(v_{\beta}\right)_{\beta \in \mathcal{B}}$ in $A$ such that $\left(v_{\beta} x_{2}\right)_{\beta \in \mathcal{B}}$ converges to $e_{A}$. Now $\left(v_{\beta} x_{2}\left(x_{1} z_{\alpha}\right)\right)_{\beta \in \mathcal{B}}$ converges to $x_{1} z_{\alpha}$ for each fixed $\alpha \in \mathcal{A}$. Since $e_{A}+U$ is a neighbourhood of $x_{1} z_{\alpha}$ for each $\alpha \geq \alpha_{0}$, there exists $\beta_{0} \in \mathcal{B}$ such that $\left(v_{\beta} z_{\alpha}\right) x_{1} x_{2} \in e_{A}+U$ for each $\alpha \geq \alpha_{0}$ and $\beta \geq \beta_{0}$. Hence, there is a net $\left(v_{\beta} z_{\alpha}\right)_{(\beta, \alpha) \in \mathcal{B} \times \mathcal{A}}$ in $A$ such that $\left(\left(v_{\beta} z_{\alpha}\right)\left(x_{1} x_{2}\right)\right)_{(\beta, \alpha) \in \mathcal{B} \times \mathcal{A}}$ converges to $e_{A}$. Consequently, $x_{1} x_{2} \in G^{t}(A)$.

Proposition 7. Let $A$ be a commutative complex unital semitopological algebra. Then

$$
\sigma_{A}^{t}(p(x))=p\left(\sigma_{A}^{t}(x)\right)
$$

for any complex non-constant polynomial $p$ and element $x \in A$.

Proof. Fix a polynomial $p$ of degree $n>0$ and $x \in A$. If $\lambda \in \sigma_{A}^{t}(x)$, then $x-\lambda e_{A} \notin G^{t}(A)$. Since $p(x)-p(\lambda) e_{A}=\left(x-\lambda e_{A}\right) r(x, \lambda)$ (where $r$ is a polynomial in two variables), we have $p(x)-p(\lambda) e_{A} \notin G^{t}(A)$ by Lemma 3 , i.e. $p(\lambda) \in \sigma_{A}^{t}(p(x))$. Thus, $p\left(\sigma_{A}^{t}(x)\right) \subset \sigma_{A}^{t}(p(x))$.

Conversely, let $\mu \in \sigma_{A}^{t}(p(x))$. Then $g(t)=p(t)-\mu=\alpha\left(t-\mu_{1}\right) \cdots\left(t-\mu_{n}\right)$, where $\alpha, \mu_{1}, \ldots, \mu_{n} \in \mathbb{C}, \alpha \neq 0$ and $\mu_{1}, \ldots, \mu_{n}$ are the zeros of $g$. It follows that $p\left(\mu_{i}\right)=\mu$ for each $1 \leq i \leq n$. Now we have the factorization $g(x)=$ $p(x)-\mu e_{A}=\alpha\left(x-\mu_{1} e_{A}\right) \cdots\left(x-\mu_{n} e_{A}\right)$. Since $\mu \in \sigma_{A}^{t}(p(x))$, it follows that $g(x) \notin G^{t}(A)$. Hence, $x-\mu_{k} e_{A} \notin G^{t}(A)$ for some $k$ by Lemma 3, i.e. $\mu_{k} \in \sigma_{A}^{t}(x)$. Therefore, $\mu=p\left(\mu_{k}\right) \in p\left(\sigma_{A}^{t}(x)\right)$. Thus $\sigma_{A}^{t}(p(x)) \subset p\left(\sigma_{A}^{t}(x)\right)$.

4.3. Functional topological spectrum. Next we describe the class of semitopological algebras, all elements of which have functional topological spectrum.

Proposition 8. Let $A$ be a commutative unital simplicial Gelfand-Mazur algebra. If $\varphi(a) \neq 0$ for each $\varphi \in \mathfrak{M}(A)\left({ }^{10}\right)$, then $a \in G^{t}(A)$.

$\left({ }^{10}\right)$ Since $A$ is a commutative unital simplicial Gelfand-Mazur algebra, $\mathfrak{M}(A)$ is not empty. 
Proof. Let $a \in A$ be such that $\varphi(a) \neq 0$ for each $\varphi \in \mathfrak{M}(A)$. Suppose that $a \notin G^{t}(A)$. Then there is a closed maximal ideal $M$ in $A$ such that $\overline{a A} \subset M$ (because $A$ is simplicial). Since $A$ is also a Gelfand-Mazur algebra, there exists $\varphi \in \mathfrak{M}(A)$ such that $M=\operatorname{ker} \varphi$. Hence, $\varphi(a)=0$, contrary to assumption.

COROLlary 9. Every element of a commutative unital simplicial Gelfand-Mazur algebra has functional topological spectrum.

Proof. Let $a \in A$ and $\lambda \in \sigma_{A}^{t}(a)$. Then $a-\lambda e_{A} \notin G^{t}(A)$. Hence, by Proposition 8 , there is a $\varphi \in \mathfrak{M}(A)$ such that $\varphi(a)=\lambda$. Thus, $\sigma_{A}^{t}(a) \subset$ $\{\varphi(x): \varphi \in \mathfrak{M}(A)\}$.

COROLlary 10. Every element of a commutative unital locally m-pseudoconvex Hausdorff algebra has functional topological spectrum.

Proof. Every commutative unital locally $m$-pseudoconvex Hausdorff algebra is a simplicial algebra (see [5, Corollary 5]) and a Gelfand-Mazur algebra (see, for example, [1, Corollary 2]). Now apply Corollary 9.

4.4. Properties of topological spectral radius. Now we list some properties of the topological spectral radius.

Proposition 9. Let $A$ be a semitopological algebra. The topological spectral radius $\varrho_{A}^{t}$ has the following properties:

(a) $\varrho_{A}^{t}(\mu x)=|\mu| \varrho_{A}^{t}(x)$ for all $x \in A$ and $\mu \in \mathbb{K}$;

(b) $\varrho_{A}^{t}(x y)=\varrho_{A}^{t}(y x)$ for all $x, y \in A$;

(c) if $A$ is commutative, then $\varrho_{A}^{t}\left(x^{n}\right)=\varrho_{A}^{t}(x)^{n}$ for all $x \in A$ and $n \in \mathbb{N}$. In particular, if every element in $A$ has functional topological spectrum, then

(d) $\varrho_{A}^{t}(x+y) \leq \varrho_{A}^{t}(x)+\varrho_{A}^{t}(y)$ for all $x, y \in A$;

(e) $\varrho_{A}^{t}(x y) \leq \varrho_{A}^{t}(x) \varrho_{A}^{t}(y)$ for all $x, y \in A$.

Proof. Let $x \in A$ be such that $\sigma_{A}^{t}(x) \neq \emptyset$, and $\mu \in \mathbb{K} \backslash\{0\}$ (the case when $\mu=0$ is trivial). Since $\sigma_{A}^{t}(\mu x)=\mu \sigma_{A}^{t}(x)$ for each $\mu \in \mathbb{K}$ by Proposition 4(a), we have $|\lambda| \leq|\mu| \varrho_{A}^{t}(x)$ for all $\lambda \in \sigma_{A}^{t}(\mu a)$ and $\mu \in \mathbb{K}$. Therefore, the relations

$$
\varrho_{A}^{t}(\mu x) \leq|\mu| \varrho_{A}^{t}(x)=|\mu| \varrho_{A}^{t}\left(\mu^{-1}(\mu x)\right) \leq \varrho_{A}^{t}(\mu x)
$$

yield (a). Statements (b) and (c) follow from Proposition 4(d) and Proposition 7 respectively. If $A$ has functional topological spectrum, then we can represent every $\lambda \in \sigma_{A}^{t}(x+y)$ in the form $\lambda=\varphi_{0}(x)+\varphi_{0}(y)$ for some $\varphi_{0} \in \mathfrak{M}(A)$. Since

$$
|\lambda| \leq\left|\varphi_{0}(x)\right|+\left|\varphi_{0}(y)\right| \leq \varrho_{A}^{t}(x)+\varrho_{A}^{t}(y),
$$

statement (d) follows. Statement (e) is proved similarly. 
Corollary 11. Let $A$ be a unital semitopological algebra. If every element of $A$ has functional topological spectrum, then the topological spectral radius is a submultiplicative seminorm on $A$.

Corollary 12. Let $A$ be a commutative unital locally $m$-pseudoconvex Hausdorff algebra. Then the topological spectral radius is a submultiplicative seminorm on $A$.

5. Open problems. Now we state several open problems, connected with topological invertibility of elements.

5.1. Let $A$ be a semitopological algebra and $x \in G^{t}(A)$. Then there exist nets $\left(x_{\alpha}\right)_{\alpha \in \mathcal{A}}$ and $\left(y_{\mu}\right)_{\mu \in \mathcal{M}}$ such that $\lim _{\alpha} x_{\alpha} x=e_{A}$ and $\lim _{\mu} x y_{\mu}=e_{A}$. Let $G_{0}^{t}(A)=\left\{x \in G^{t}(A)\right.$ : there exists a net $\left(x_{\alpha}\right)_{\alpha \in \mathcal{A}}$ such that $\lim _{\alpha} x_{\alpha} x=e_{A}$ and $\left.\lim _{\alpha} x x_{\alpha}=e_{A}\right\}$. If $A$ is commutative, then $G_{0}^{t}(A)=G^{t}(A)$.

Problem 1. Does there exist a non-commutative semitopological algebra $A$ for which $G_{0}^{t}(A)=G^{t}(A)$ ?

5.2. By Corollary 2 , if $A$ is a unital Fréchet algebra with discontinuous inversion $x \mapsto x^{-1}$, then $G^{t}(A) \backslash G(A) \neq \emptyset$, but the converse is open so far.

Problem 2. Let $A$ be a unital Fréchet algebra. Does the discontinuity of the inversion $x \mapsto x^{-1}$ on $G(A)$ follow from $G^{t}(A) \backslash G(A) \neq \emptyset$ ?

5.3. By [18, Theorem 2], there is a semitopological algebra $A$ with $\overline{G^{t}(A)}$ $=A$.

Problem 3. For which semitopological algebras $A$ does $\overline{G^{t}(A)}=A$ hold?

Problem 4. For which semitopological algebras $A$ does $\overline{G(A)}=G^{t}(A)$ hold?

5.4. We have the following result.

Proposition 10. A semitopological algebra $A$ is a $Q$-algebra $\left({ }^{11}\right)$ if and only if $G^{t}(A)$ is open and $\overline{\sigma_{A}^{t}(a)}=\sigma_{A}(a)$ for each $a \in A \backslash G(A)$.

Proof. If $A$ is a $Q$-algebra, then $G^{t}(A)=G(A)$. Therefore, $G^{t}(A)$ is open in $A$ and $\overline{\sigma_{A}^{t}(a)}=\sigma_{A}(a)$ for each $a \in A \backslash G(A)$.

Conversely, let $A$ be a semitopological algebra such that $G^{t}(A)$ is open and $\overline{\sigma_{A}^{t}(a)}=\sigma_{A}(a)$ for each $a \in A \backslash G(A)$. If now $a \in A \backslash G(A)$, then $0 \in \sigma_{A}(a)$. Therefore there exists a sequence $\left(\lambda_{n}\right)$ in $\sigma_{A}^{t}(a)$ which converges to 0 . Since $a-\lambda_{n} e_{A} \in A \backslash G^{t}(A)$ and $A \backslash G^{t}(A)$ is closed in $A$, we have $a \in A \backslash G^{t}(A)$. Thus, $G(A)=G^{t}(A)$. Consequently, $A$ is a $Q$-algebra.

Problem 5. Describe the elements of a semitopological algebra $A$ for which $\overline{\sigma_{A}^{t}(a)}=\sigma_{A}(a)$.

$\left({ }^{11}\right)$ That is, $G(A)$ is open in $A$. 
5.5. If $A$ is an algebra and $B$ a maximal commutative subalgebra of $A$, then $\sigma_{A}(a)=\sigma_{B}(a)$. It is not clear whether a similar result holds for the topological spectrum.

Problem 6. Let $A$ be a semitopological algebra. Does there exist a maximal commutative subalgebra such that $\sigma_{A}^{t}(a) \neq \sigma_{B}^{t}(a)$ for some $a \in A$ ?

\section{References}

[1] M. Abel, Gelfand-Mazur algebras, in: Topological Vector Spaces, Algebras and Related Areas (Hamilton, ON, 1994), Longman, Harlow, 1994, 116-129.

[2] - Advertive topological algebras, in: General Topological Algebras (Tartu, 1999), Math. Stud. (Tartu) 1, Est. Math. Soc., Tartu, 2001, 14-24.

[3] -, Descriptions of the topological radical in topological algebras, ibid., 25-31.

[4] - Survey of results on Gelfand-Mazur algebras, in: Non-Normed Topological Algebras (Rabat, 2000), E. N. S. Takaddoum Publ., Rabat, 2004, 14-25.

[5] -, Inductive limits of Gelfand-Mazur algebras, Int. J. Pure Appl. Math. 16 (2004), 363-378.

[6] M. Akkar, A. Beddaa et M. Oudadess, Sur une classe d'algèbres topologiques, Bull. Belg. Math. Soc. Simon Stevin 3 (1996), 13-24.

[7] - - - - , Topologically invertible elements in metrizable algebras, Indian J. Pure Appl. Math. 27 (1996), 1-5.

[8] R. F. Arens, The space $L^{\omega}$ and convex topological rings, Bull. Amer. Math. Soc. 52 (1946), 931-935.

[9] H. Arizmendi, A. Carillo and L. Palacios, On $Q_{t}$-algebras, manuscript.

[10] V. K. Balachandran, Topological Algebras, North-Holland Math. Stud. 185, NorthHolland, Amsterdam, 2000.

[11] S. J. Batt and A. D. Thatte, On topological invertibility, Indian J. Pure Appl. Math. 15 (1984), 1308-1312.

[12] B. Beckenstein, L. Narici and Ch. Suffel, Topological Algebras, North-Holland Math. Stud. 24, North-Holland, Amsterdam, 1977.

[13] A. Beddaa, Algèbres localement convexes advertiblement complètes et continuité automatique de morphismes, Thèse Sci. Math., Univ. Mohamed V, Rabat, 1997.

[14] A. Białynicki-Birula and W. Żelazko, On the multiplicative-linear functionals on the Cartesian product of algebras, Bull. Acad. Polon. Sci. Sér. Sci. Math. Astronom. Phys. 5 (1957), 201-203.

[15] R. Choukri, A. El Kinani et M. Oudadess, Inversibilité topologique et problème de l'idéal fermé, Bol. Soc. Mat. Mexicana (3) 9 (2003), 109-117.

[16] K. Luha, Topological invertibility in topological algebras, Tartu Ül. Toimetised 940 (1992), 71-74.

[17] W. Żelazko, Selected Topics in Topological Algebras, Aarhus Univ. Lecture Notes 31, Aarhus Univ., Aarhus, 1971.

[18] - Topological simplicity of a certain $\mathcal{L} \mathcal{F}$-algebra, Period. Math. Hungar. 35 (1997), $145-148$.

[19] - When a commutative unital F-algebra has a dense principal ideal, in: Topological Algebras and Their Applications, Contemp. Math. 341, Amer. Math. Soc., Providence, RI, 2004, 133-137.

[20] - F-algebras: some results and open problems, in: Functional Analysis and its Applications, North-Holland Math. Stud. 197, Elsevier, Amsterdam, 2004, 317-326. 
[21] W. Żelazko, Continuous characters and joint topological spectrum, submitted.

Mati Abel

Institute of Pure Mathematics

University of Tartu

Liivi 2-614, 50409 Tartu, Estonia

E-mail: mati.abel@ut.ee
Wiesław Żelazko Institute of Mathematics Polish Academy of Sciences P.O. Box 137, Śniadeckich 8 00-956 Warszawa, Poland E-mail: zelazko@impan.gov.pl

Received June 30, 2006 